\title{
Exact pricing and large-time asymptotics for the modified SABR model and the Brownian exponential functional
}

\author{
Martin Forde*†
}

November 18, 2010

\begin{abstract}
We derive a closed-form expression for the stock price density under the modified SABR model (see section 2.4 in Islah[Isl09]) with zero correlation, for $\beta=1$ and $\beta<1$, using the known density for the Brownian exponential functional for $\mu=0$ given in Matsumoto\&Yor[MY05], and then reversing the order of integration using Fubini's theorem. We then derive a large-time asymptotic expansion for the Brownian exponential functional for $\mu=0$, and we use this to characterize the large-time behaviour of the stock price distribution for the modified SABR model; the asymptotic stock price "density" is just the transition density $p\left(t, S_{0}, S\right)$ for the CEV process, integrated over the large-time asymptotic "density" $\frac{1}{t} e^{-1 / 2 t}$ associated with the Brownian exponential functional (re-scaled), as we might expect. We also compute the large-time asymptotic behaviour for the price of a call option, and we show precisely how the implied volatility tends to zero as the maturity tends to infinity, for $\beta=1$ and $\beta<1$. These results are shown to be consistent with the general large-time asymptotic estimate for implied variance given in Tehranchi[Tehr09]. The modified SABR model is significantly more tractable than the standard SABR model. Moreover, the integrated variance for the modified model is infinite a.s. as $t \rightarrow \infty$, in contrast to the standard SABR model, so in this sense the modified model is also more realistic.
\end{abstract}

\section{Introduction}

Throughout this paper we let $B=\left\{B_{t}, t \geq 0\right\}$ denote a one-dimensional Brownian motion started at zero defined on a probability space $(\Omega, \mathcal{F}, \mathbb{P})$, and $B_{t}^{(\mu}=\left\{B_{t}+\mu t, t \geq 0\right\}$ denote the corresponding Brownian motion with constant drift $\mu \in \mathbb{R}$. We consider the exponential functional

$$
A_{t}^{(\mu)}=\int_{0}^{t} e^{2 B_{s}^{(\mu)}} d s
$$

which is the time-integral of geometric Brownian motion. This additive functional plays an important role in several domains, e.g. in pricing arithmetic Asian call options, as the time-change for the SABR and Hull-White stochastic volatility models, and for Brownian motion on the upper half plane under the hyperbolic metric. This fact motivates detailed studies about this functional.

In this article, we only consider the case $\mu=0$, for which the analysis is a lot cleaner. At fixed finite time, only an integral expression is known for the density of $A_{t}=A_{t}^{(\mu)}$. This is obtained as a by-product of the well known Bougerol identity, which states that $W_{A_{t}} \stackrel{d}{=} \sinh \left(W_{t}\right)$, where $\left(W_{t}\right)$ is another Brownian motion, independent of $B$ (see [AG97],[CMY98],[MY05] for details). Gulisashvili\&Stein[GS06],[GS07] derive sharp tail estimates for $A_{t}^{\mu}$ use saddlepoint methods, and they use this to derive tail estimates for the stock price under the Hull-White stochastic volatility model, and arithmetic Asian call options under the Black-Scholes model. $A_{t}^{\mu}$ is also the time-change for the SABR and Hull-White stochastic volatility models with zero correlation. The small-time asymptotics for the SABR model are well documented (see e.g. [Forde09], [HL09], [Laur08]).

In section 2 , we introduce the modified uncorrelated SABR model for $\beta=1$ as a stochastic volatility model with the same law as an arithmetic Brownian motion evaluated at an independent time given by the Brownian exponential functional. The modified model corresponds to $\mu=0$ (and for $\mu=0$ Bougerol's identity holds); for this reason the modified model is far more tractable then the standard SABR model for $\rho=0$. Using Fubini's theoerem, we derive

*Department of Mathematical Sciences, Dublin City University, Glasnevin, Dublin 9, Ireland (Martin.Forde@dcu.ie), work supported by SFI grant for the Edgeworth Centre for Financial Mathematics.

${ }^{\dagger}$ The author would like to thank Alan Lewis for many valuable insights. 
a semi-closed form expression for the log stock price density by performing the inner integration analytically. We perform a similar analysis for $\beta<1$ by integrating the transition density for the CEV process over the density of $A_{t}$ and then using Fubini's theorem. In section 3 we concentrate on large-time asymptotics; we first derive a full asymptotic expansion for the density of $A_{t}$ which sharpens a result in Matsumoto\&Yor[MY05] where the leading order term is given. Using the dominated convergence theorem, we then characterize the large-time asymptotic behaviour of the stock price density at leading order for the modified SABR model with $\beta=1$ and $\beta<1$. In both cases we find that the re-scaled asymptotic "density" is not a true density function, and is obtained by integrating the density of arithmetic Brownian motion (respectively the CEV process) over the asymptotic re-scaled "density" for $A_{t}$, as we might expect. Using an alternate representation for the price of a call option, we also derive large-time asymptotics for call options prices, and we show precisely how the implied volatility for a fixed-strike put or call option tends to zero as the maturity tends to infinity; specifically, we find that the dimensionless implied variance is $O(\log t)$ as $t \rightarrow \infty$. We show that these large-time estimates for implied volatility are consistent with the main result (Theorem 3.1) in Tehranchi[Tehr09]. In particular, we see that the large-time behaviour for call options and implied volatility is qualitatively different to the large-time asymptotics obtained for the Heston model in Forde,Jacquier\&Mijatovic[FJM09] using Laplace's method for contour integrals. For the Heston model, the difference between the initial stock price and the call price is exponentially small as $t \rightarrow \infty$, and the implied volatility tends to a constant; for the modified uncorrelated SABR model, the difference is $O\left(t^{-\frac{1}{2}}\right)$ and the implied volatility tends to zero.

\section{The modified SABR model}

\subsection{The case $\beta=1$}

We first consider the modified uncorrelated SABR model defined in section 2.4 in [Isl09] for $\beta=1$

$$
\left\{\begin{array}{l}
d X_{t}=-\frac{1}{2} Y_{t}^{2} d t+Y_{t} d W_{t} \\
d Y_{t}=\frac{1}{2} \alpha^{2} Y_{t} d t+\alpha Y_{t} d B_{t}
\end{array}\right.
$$

where $X_{t}=\log S_{t}$ is a $\log$ stock price process, $X_{0}=x_{0}, Y_{0}=y_{0}>0$, and $W$ and $B$ are two independent Brownian motions. Note that $Y_{t}=y_{0} e^{\alpha B_{t}}$. This model is a special case of the well known Hull-White stochastic volatility model (see Gulisashvili\&Stein[GS06]). From the scaling property of Brownian motion we see that the integrated variance $\int_{0}^{t} Y_{s}^{2} d s$ satisfies

$$
\int_{0}^{t} Y_{s}^{2} d s=y_{0}^{2} \int_{0}^{t} e^{2 \alpha B_{s}} d s \stackrel{d}{=} \frac{y_{0}^{2}}{\alpha^{2}} \int_{0}^{\alpha^{2} t} e^{2 B_{u}} d u
$$

and the density of $A_{t}=\int_{0}^{t} e^{2 B_{s}} d s$ is obtained as a by-product of Bougerol's identity ${ }^{1}$

$$
f(u, t)=\frac{1}{\sqrt{2 \pi u^{3}}} \frac{1}{\sqrt{2 \pi t}} \int_{-\infty}^{\infty} \cosh (\xi) e^{-(\cosh \xi)^{2} / 2 u-(\xi+i \pi / 2)^{2} / 2 t} d \xi
$$

(see Theorem 4.4 in [MY05]).

Proposition 2.1 The density $p_{t}(x)$ of $X_{t}-x_{0}$ is given by

$$
p_{t}(x)=\frac{\sigma e^{-\frac{1}{2} x}}{\sqrt{2 \pi \alpha^{2} t}} \int_{-\infty}^{\infty} \cosh (\xi) \frac{K_{1}\left(\frac{1}{2} \sqrt{x^{2}+\sigma^{2}(\cosh \xi)^{2}}\right)}{2 \pi \sqrt{\left(x^{2}+\sigma^{2}(\cosh \xi)^{2}\right)}} e^{-(\xi+i \pi / 2)^{2} / 2 \alpha^{2} t} d \xi,
$$

where

$$
\sigma=y_{0} / \alpha
$$

and $K_{1}($.$) is the modified Bessel function of the second kind of order 1$ (see page 374 in Abramowitz\&Stegun[AS72] for a definition).

Proof. $X_{t}$ is identical in law to an arithmetic Brownian motion with volatility $\sigma$ and drift $-\frac{1}{2} \sigma^{2}$ evaluated at the independent time $A_{\alpha^{2} t}$. Using this relation, we can compute the density $p_{t}(x)$ of $X_{t}-x_{0}$ as

$$
\begin{aligned}
p_{t}(x) & =\int_{0}^{\infty} \frac{1}{\sigma \sqrt{2 \pi u}} e^{-\left(x+\frac{1}{2} \sigma^{2} u\right)^{2} / 2 \sigma^{2} u} f\left(u, \alpha^{2} t\right) d u \\
& =\int_{0}^{\infty} \frac{1}{\sigma \sqrt{2 \pi u}} e^{-\left(x+\frac{1}{2} \sigma^{2} u\right)^{2} / 2 \sigma^{2} u} \frac{1}{\sqrt{2 \pi u^{3}}} \frac{1}{\sqrt{2 \pi \alpha^{2} t}} \int_{-\infty}^{\infty} \cosh (\xi) e^{-(\cosh \xi)^{2} / 2 u-(\xi+i \pi / 2)^{2} / 2 \alpha^{2} t} d \xi d u
\end{aligned}
$$

\footnotetext{
${ }^{1}$ Bougerol's identity states that $\int_{0}^{t} e^{B_{s}} d W_{s}, \sinh \left(B_{t}\right)$ and $W_{A_{t}}$ are identical in law; note that this is only valid for driftless Brownian motion i.e. $\mu=0$.
} 


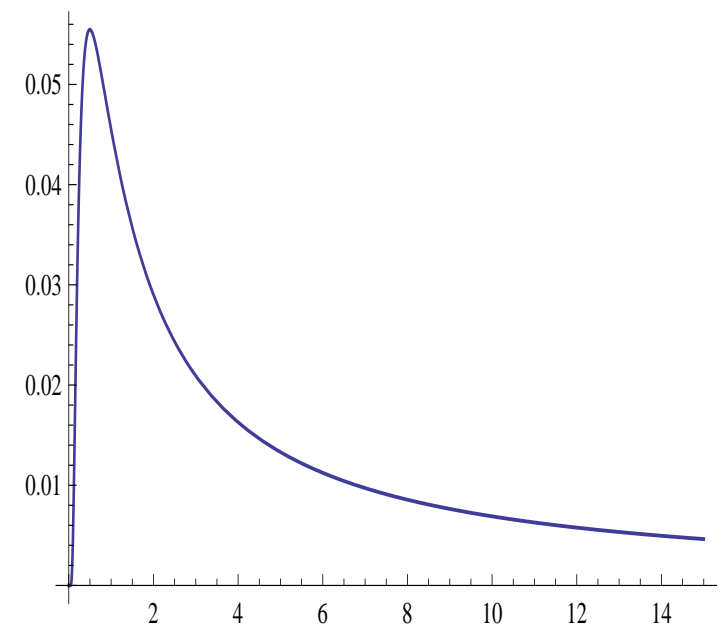

Figure 1: Here we have plotted the density of $A_{t}$ for $t=1$.

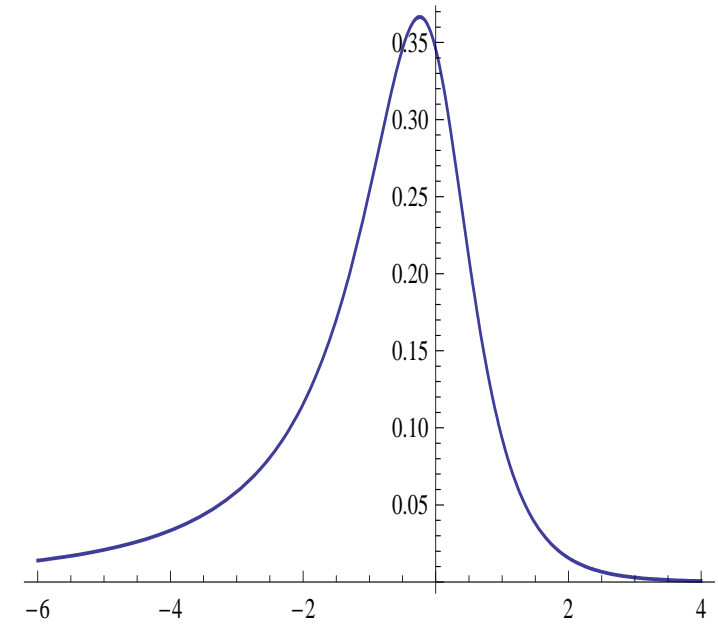

Figure 2: Here we have plotted the $\log$ Stock price density for the modified SABR model with $S_{0}=1, y_{0}=1, \alpha=$ $1, \beta=1$.

Using Fubini's theorem to interchange the order of integration ${ }^{2}$ and evaluating the integral over $u$ analytically, we obtain (2).

Remark 2.1 We have effectively removed a dimension of integration in the formula in [Isl09] for the case for the modified SABR model with $\beta=1$.

\subsection{The case $\beta<1$}

The constant elasticity of variance $(\mathrm{CEV})$ diffusion process of Cox[Cox75] is defined by the SDE

$$
d S_{t}=\delta S_{t}^{\beta} d W_{t}
$$

with $\beta \in(0,1), \delta>0$. The origin is an exit boundary for $\beta \in\left(\frac{1}{2}, 1\right)$, and a regular boundary for $\beta \leq \frac{1}{2}$, which we specify as absorbing to ensure that $\left(S_{t}\right)$ is a martingale. Infinity is a natural, non-attracting boundary. The transition density for the CEV process is given by

$$
p\left(t, S_{0}, S\right)=\frac{S^{-2 \bar{\beta}-\frac{3}{2}} S_{0}^{\frac{1}{2}}}{\delta^{2}|\bar{\beta}| t} \exp \left(-\frac{S_{0}^{-2 \bar{\beta}}+S^{-2 \bar{\beta}}}{2 \delta^{2} \bar{\beta}^{2} t}\right) I_{\nu}\left(\frac{S_{0}^{-\bar{\beta}} S^{-\bar{\beta}}}{\delta^{2} \bar{\beta}^{2} t}\right) \quad(S>0),
$$

\footnotetext{
${ }^{2}$ see Appendix A for justification.
} 
where $\bar{\beta}=\beta-1, \nu=\frac{1}{2 \mid \bar{\beta}} \mid$, and $I_{\nu}($.$) is the modified Bessel function of the first kind (see page 374$ in Abramowitz\&Stegun[AS72] for a definition, and also Davydov\&Linetsky[DavLin01]). Combining the CEV model with uncorrelated stochastic volatility, the modified SABR model is defined by the stochastic differential equations

$$
\left\{\begin{array}{l}
d S_{t}=S_{t}^{\beta} Y_{t} d W_{t} \\
d Y_{t}=\frac{1}{2} \alpha^{2} Y_{t} d t+\alpha Y_{t} d B_{t}
\end{array}\right.
$$

with $d W_{t} d B_{t}=0, Y_{0}=y_{0}>0$. Equivalently, we can write the dynamics of the model as

$$
\left\{\begin{array}{l}
d S_{t}=\delta S_{t}^{\beta} Y_{t} d W_{t} \\
d Y_{t}=\frac{1}{2} \alpha^{2} Y_{t} d t+\alpha Y_{t} d B_{t}
\end{array}\right.
$$

with $Y_{0}=1, \delta=y_{0}$.

Proposition 2.2 The density $p_{t}(S)$ of $S_{t}$ is the CEV transition density integrated over the distribution of the integrated variance for $y_{0}=1$ :

$p_{t}(S)=\frac{A}{\sqrt{2 \pi \alpha^{2} t}} \int_{-\infty}^{\infty} \cosh (\xi)\left[c_{12} F_{1}\left(\frac{3}{4}-\frac{1}{4} \gamma, \frac{1}{4}(3+\gamma), \frac{1}{2}, \frac{g(\xi)^{2}}{4 z_{0}^{2} z^{2}}\right)+c_{2} g(\xi){ }_{2} F_{1}\left(\frac{5}{4}-\frac{1}{4} \gamma, \frac{1}{4}(5+\gamma), \frac{3}{2}, \frac{g(\xi)^{2}}{4 z_{0}^{2} z^{2}}\right)\right] \cdot e^{-\frac{(\xi+i \pi / 2)^{2}}{2 \alpha^{2} t}} d \xi$,

where $\gamma=\frac{1}{|\beta|}$ and

$$
\begin{aligned}
A & =\bar{\beta}^{2} \delta \sqrt{S_{0} z_{0} z}\left[\sqrt{\pi} S^{\frac{3}{2}} \Gamma\left(\frac{1}{4}(-1+\gamma)\right) \Gamma\left(\frac{1}{4}(1+\gamma)\right) z^{2}\right]^{-1}, \\
g(\xi) & =z_{0}^{2}+\left(1+z_{0}^{2} \bar{\beta}^{2} \delta^{2}(\cosh \xi)^{2}\right) z^{2}, \\
c_{1} & =-z_{0} z \Gamma\left(\frac{1}{4}(\gamma-1)\right) \Gamma\left(\frac{1}{4}(3+\gamma)\right) \sin \left(\frac{1}{4} \pi(\gamma+1)\right), \\
c_{2} & =\Gamma\left(\frac{1}{4}(1+\gamma)\right) \Gamma\left(\frac{1}{4}(5+\gamma)\right) \sin \left(\frac{1}{4} \pi(1-\gamma)\right)
\end{aligned}
$$

and $_{2} F_{1}(a, b, c, z)$ is the hypergeometric function (see page 556 in AbramowitzBSStegun[AS72] for a definition), $\delta=y_{0} / \alpha$, $z=S^{\bar{\beta}}, z_{0}=S_{0}^{\bar{\beta}}$.

Proof. $S_{t}$ is identical in law to the CEV process with $\delta=y_{0} / \alpha$ evaluated at the independent time $A_{\alpha^{2} t}$. Using this relation, we can compute the density $p_{t}(S)$ of $S_{t}$ by setting $\delta=y_{0} / \alpha$ and then computing

$$
\begin{aligned}
p_{t}(S) & =\int_{0}^{\infty} p\left(u, S_{0}, S\right) f\left(u, \alpha^{2} t\right) d u \\
& =\int_{0}^{\infty} p\left(u, S_{0}, S\right) \frac{1}{\sqrt{2 \pi u^{3}}} \frac{1}{\sqrt{2 \pi \alpha^{2} t}} \int_{-\infty}^{\infty} \cosh (\xi) e^{-(\cosh \xi)^{2} / 2 u-(\xi+i \pi / 2)^{2} / 2 \alpha^{2} t} d \xi d u .
\end{aligned}
$$

Using Fubini's theorem to interchange the order of integration ${ }^{3}$ and evaluating the integral over $u$ analytically, we obtain (7).

Corollary 2.3 To price call options we just integrate the call payoff $(S-K)^{+}$over the density $p_{t}(S)$ in $(7)$

$$
\mathbb{E}\left(S_{t}-K\right)^{+}=\int_{0}^{\infty}(S-K)^{+} p_{t}(S) d S .
$$

Remark 2.2 The main qualitative difference between the SABR model and the modified SABR model is that $A_{\infty}=\infty$ a.s. for the modified case because the driftless Brownian motion $B$ is recurrent, but for the usual SABR model, the associated exponential functional is $\int_{0}^{t} e^{2 B_{s}^{(\mu)}} d s$ for $\mu=-\frac{1}{2}$, which is finite a.s. (see [MY05]), so in this sense the modified SABR model is more realistic.

\footnotetext{
${ }^{3}$ see Appendix B for justification.
} 


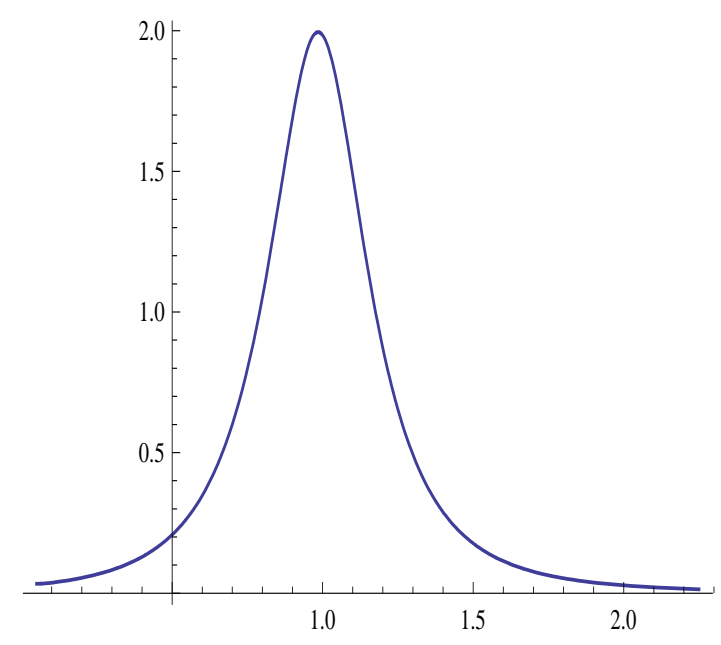

Figure 3: Here we have plotted the Stock price density for the modified SABR model with $S_{0}=1, y_{0}=1, \alpha=1, \beta=$ $.5, \delta=.2$. Note that the density integrates to less than one as there is a non-zero probability of absorption.

\subsection{Probability of absorption at zero for $\beta<1$}

For the standard CEV model in (4), from e.g. page 312 in Lewis[Lew00], we have the following well known expression for the probability of absorption at $S=0$ by time $t$

$$
\mathbb{P}\left(S_{t}=0\right)=\frac{1}{\Gamma(\nu)} G\left(\nu, \frac{\frac{1}{2} S_{0}^{-2 \bar{\beta}}}{\delta^{2} \beta^{2} t}\right),
$$

where $\nu=\frac{1}{2(1-\beta)}>0$ and $G(\nu, x)=\int_{x}^{\infty} t^{\nu-1} e^{-t} d t$ is the complementary incomplete Gamma function. The probability of absorption for the modified uncorrelated SABR model is then obtained by integrating $\mathbb{P}\left(S_{t}=0\right)$ with $\delta=y_{0} / \alpha$ over the density of $A_{\alpha^{2} t}$ as

$$
\begin{aligned}
& \int_{0}^{\infty} \frac{1}{\Gamma(\nu)} G\left(\nu, \frac{\frac{1}{2} S_{0}^{-2 \bar{\beta}} \alpha^{2}}{y_{0}^{2} \beta^{2} u}\right) f\left(u, \alpha^{2} t\right) d u \\
= & \int_{0}^{\infty} \frac{1}{\Gamma(\nu)} G\left(\nu, \frac{\frac{1}{2} S_{0}^{-2 \bar{\beta}} \alpha^{2}}{y_{0}^{2} \beta^{2} u}\right) \frac{1}{\sqrt{2 \pi u^{3}}} \frac{1}{\sqrt{2 \pi \alpha^{2} t}} \int_{-\infty}^{\infty} \cosh (\xi) e^{-(\cosh \xi)^{2} / 2 u-(\xi+i \pi / 2)^{2} / 2 \alpha^{2} t} d \xi d u .
\end{aligned}
$$

\section{Large-time asymptotics}

\subsection{The Brownian exponential functional}

We first summarize some interesting results in Matsumoto\&Yor[MY05]. Letting $t \rightarrow \infty$ in (1) and using the dominated convergence theorem, we have the following large-time behaviour for the distribution of $A_{t}$

$$
\lim _{t \rightarrow \infty} \sqrt{2 \pi t} \mathbb{P}\left(A_{t} \in d u\right)=\frac{1}{\sqrt{2 \pi u^{3}}} \int_{-\infty}^{\infty} \lim _{t \rightarrow \infty}\left[\cosh (\xi) e^{-(\cosh \xi)^{2} / 2 u-(\xi+i \pi / 2)^{2} / 2 t}\right] d \xi=\frac{1}{u} e^{-1 / 2 u} d u \quad(u>0),
$$

where we have used the identity ${ }^{4}$

$$
\frac{1}{\sqrt{2 \pi u^{3}}} \int_{-\infty}^{\infty} \cosh (\xi) e^{-(\cosh \xi)^{2} / 2 u} d \xi=\frac{1}{\sqrt{2 \pi u^{3}}}\left(\int_{-\infty}^{-1}+\int_{1}^{\infty}\right) \frac{b}{\sqrt{b^{2}-1}} e^{-b^{2} / 2 u} d \xi=\frac{1}{u} e^{-1 / 2 u} .
$$

(see Theorem 4.4 in [MY05]). Note that the right-hand side of (11) integrates to infinity, so it is not a probability density function.

By the scaling property of Brownian motion

$$
A_{t} \stackrel{d}{=} t \int_{0}^{1} e^{2 \sqrt{t} B_{s}} d s
$$

\footnotetext{
${ }^{4}$ This identity can be proved using the same double integral trick with polar coordinates that we use to prove that the standard Normal distribution integrates to 1 .
} 
and (using Laplace's method)

$$
\frac{1}{\sqrt{t}} \log \int_{0}^{1} e^{2 \sqrt{t} B_{s}} d s \rightarrow 2 \max _{0 \leq s \leq t} B_{s}
$$

and $\frac{1}{\sqrt{t}} \log \left(A_{t}\right)$ converges in law to $2|Z|$ as $t \rightarrow \infty$ where $Z$ is a standard Normal r.v. (see Proposition 4.6 in [MY05]).

We now prove the a large-time asymptotic expansion for the density of $A_{t}$ which sharpens the result in (11) in [MY05]:

Proposition 3.1 We have the following expansion for the density of $A_{t}$

$$
\sqrt{2 \pi t} \mathbb{P}\left(A_{t} \in d u\right)=\left[\frac{1}{u} e^{-1 / 2 u}+\frac{1}{\sqrt{2 \pi u^{3}}} \sum_{n=1}^{\infty} \frac{1}{n !}(-1)^{n} \int_{-\infty}^{\infty} \cosh (\xi) e^{-(\cosh \xi)^{2} / 2 u}\left[\frac{(\xi+i \pi / 2)^{2}}{2 t}\right]^{n} d \xi\right] d u .
$$

Proof. Recall that

$$
f(u, t)=\frac{1}{\sqrt{2 \pi u^{3}}} \frac{1}{\sqrt{2 \pi t}} \int_{-\infty}^{\infty} \cosh (\xi) e^{-(\cosh \xi)^{2} / 2 u-(\xi+i \pi / 2)^{2} / 2 t} d \xi .
$$

Expanding the exponential we have

$$
\sqrt{2 \pi t} \mathbb{P}\left(A_{t} \in d u\right)=\frac{d u}{\sqrt{2 \pi u^{3}}} \int_{-\infty}^{\infty} \cosh (\xi) e^{-(\cosh \xi)^{2} / 2 u} \sum_{n=0}^{\infty} \frac{1}{n !}(-1)^{n}\left[\frac{(\xi+i \pi / 2)^{2}}{2 t}\right]^{n} d \xi
$$

But

$$
\begin{aligned}
& \frac{1}{\sqrt{2 \pi u^{3}}} \int_{-\infty}^{\infty} \cosh (\xi) e^{-(\cosh \xi)^{2} / 2 u} \sum_{n=0}^{\infty} \frac{1}{n !}\left|\frac{(\xi+i \pi / 2)^{2}}{2 t}\right|^{n} d \xi \\
= & \frac{1}{\sqrt{2 \pi u^{3}}} \int_{-\infty}^{\infty} \cosh (\xi) e^{-(\cosh \xi)^{2} / 2 u} \sum_{n=0}^{\infty} \frac{1}{n !}\left(\frac{\left[\left(\xi^{2}-\frac{1}{4} \pi^{2}\right)^{2}+\pi^{2} \xi^{2}\right]^{\frac{1}{2}}}{2 t}\right)^{n} d \xi \\
= & \frac{1}{\sqrt{2 \pi u^{3}}} \int_{-\infty}^{\infty} \cosh (\xi) e^{-(\cosh \xi)^{2} / 2 u} e^{\left[\left(\xi^{2}-\frac{1}{4} \pi^{2}\right)^{2}+\pi^{2} \xi^{2}\right]^{\frac{1}{2}} / 2 t} d \xi<\infty .
\end{aligned}
$$

Thus we can use Fubini's theorem to interchange the order of integration in (15) and we obtain the leading term explicitly from (12)

\subsection{The modified SABR model: $\beta=1$}

\subsubsection{The log stock price density}

Proposition 3.2 For the modified SABR model with $\beta=1$ in (2), we have the following large-time asymptotic behaviour for the density $p_{t}(x)$ of the log return $X_{t}-x_{0}$

$$
V(x, 1)=\lim _{t \rightarrow \infty} \sqrt{2 \pi \alpha^{2} t} p_{t}(x)=\frac{1}{\sqrt{x^{2}+\sigma^{2}}} e^{-\frac{1}{2}\left(x+\sqrt{x^{2}+\sigma^{2}}\right)} .
$$

Proof. Multiplying the density of $X_{t}-x_{0}$ by $\sqrt{2 \pi \alpha^{2} t}$ we obtain

$$
\sqrt{2 \pi \alpha^{2} t} p_{t}(x)=\int_{0}^{\infty} \frac{1}{\sigma \sqrt{2 \pi u}} e^{-\left(x+\frac{1}{2} \sigma^{2} u\right)^{2} / 2 \sigma^{2} u} \frac{1}{\sqrt{2 \pi u^{3}}} \int_{-\infty}^{\infty} \cosh (\xi) e^{-(\cosh \xi)^{2} / 2 u-(\xi+i \pi / 2)^{2} / 2 \alpha^{2} t} d \xi d u
$$

Letting $t \rightarrow \infty$ and applying the dominated convergence theorem we obtain

$$
\begin{aligned}
V(x, 1) & =\lim _{t \rightarrow \infty} \sqrt{2 \pi \alpha^{2} t} p_{t}(x) \\
& =\int_{0}^{\infty} \frac{1}{\sigma \sqrt{2 \pi u}} e^{-\left(x+\frac{1}{2} u \sigma^{2}\right)^{2} / 2 u \sigma} \frac{1}{\sqrt{2 \pi u^{3}}} \int_{-\infty}^{\infty} \lim _{t \rightarrow \infty}\left[\cosh (\xi) e^{-(\cosh \xi)^{2} / 2 u-(\xi+i \pi / 2)^{2} / 2 \alpha^{2} t}\right] d \xi d u \\
& =\int_{0}^{\infty} \frac{1}{\sigma \sqrt{2 \pi u}} e^{-\left(x+\frac{1}{2} u \sigma^{2}\right)^{2} / 2 u \sigma} \frac{1}{u} e^{-1 / 2 u} d u \\
& =\frac{1}{\sqrt{x^{2}+\sigma^{2}}} e^{-\frac{1}{2}\left(x+\sqrt{x^{2}+\sigma^{2}}\right)}
\end{aligned}
$$

where we have also used the identity in (12). 


\subsubsection{European and digital call options}

Proposition 3.3 For the modified SABR model with $\beta=1$ in (2), we have the following large-time asymptotic behaviour for digital and European call options on $X_{t}-x_{0}$

$$
\begin{aligned}
\lim _{t \rightarrow \infty} \sqrt{2 \pi \alpha^{2} t} \mathbb{P}\left(X_{t}-x_{0}>x\right)=\int_{x}^{\infty} \frac{1}{\sqrt{y^{2}+\sigma^{2}}} e^{-\frac{1}{2}\left(y+\sqrt{y^{2}+\sigma^{2}}\right)} d y & =E_{1}\left(\frac{1}{2}\left(x+\sqrt{x^{2}+\sigma^{2}}\right)\right)<\infty \\
F(k, 1)=\lim _{t \rightarrow \infty} \sqrt{2 \pi \alpha^{2} t}\left[1-\frac{1}{S_{0}} \mathbb{E}\left(S_{t}-K\right)^{+}\right] & =\int_{-\infty}^{\infty} \frac{e^{y} \wedge e^{k}}{\sqrt{y^{2}+\sigma^{2}} e^{-\frac{1}{2}\left(y+\sqrt{y^{2}+\sigma^{2}}\right)} d y} \\
& =E_{1}\left(\frac{1}{2}\left(-k+\sqrt{k^{2}+\sigma^{2}}\right)\right)+e^{k} E_{1}\left(\frac{1}{2}\left(k+\sqrt{k^{2}+\sigma^{2}}\right)\right) \\
& <\infty
\end{aligned}
$$

where $K=S_{0} e^{k}>0$ and $E_{1}(z)=\int_{x}^{\infty} \frac{e^{-z}}{z} d z$.

Remark 3.1 It is interesting to compare (21) against the following known result for the Heston model

$$
-\lim _{t \rightarrow \infty} \frac{1}{t} \log \left(S_{0}-\mathbb{E}\left(S_{t}-K\right)^{+}\right)=V^{*}(0)>0
$$

for some rate function $V^{*}($.$) (see Corollary 2.9$ in Forde\&Jacquier[FJ09]). For the Heston model $S_{0}-\mathbb{E}\left(S_{t}-K\right)^{+}$is exponentially small as $t \rightarrow \infty$, but for the modified SABR model with $\beta=1$, we see that $S_{0}-\mathbb{E}\left(S_{t}-K\right)^{+}=O\left(t^{-\frac{1}{2}}\right)$.

Proof. For the first result in (20), we note from (18) that

$$
\sqrt{2 \pi \alpha^{2} t} \mathbb{P}\left(X_{t}>x\right)=\int_{x}^{\infty} \int_{0}^{\infty} \frac{1}{\sigma \sqrt{2 \pi u}} e^{-\left(y+\frac{1}{2} \sigma^{2} u\right)^{2} / 2 \sigma^{2} u} \frac{1}{\sqrt{2 \pi u^{3}}} \int_{-\infty}^{\infty} \cosh (\xi) e^{-(\cosh \xi)^{2} / 2 u-(\xi+i \pi / 2)^{2} / 2 \alpha^{2} t} d \xi d u d y
$$

We then just apply the dominated convergence theorem. To obtain the final answer in terms of the $E_{1}($.$) function, we$ change the variable of integration to $z=\frac{1}{2}\left(y+\sqrt{y^{2}+\sigma^{2}}\right)$.

For the second result in $(21)$, we first note that

$$
\mathbb{E}\left(S_{t}-K\right)^{+}=\mathbb{E}\left(S_{t}\right)-\mathbb{E}\left(S_{t} \wedge K\right)=S_{0}-\mathbb{E}\left(S_{t} \wedge K\right)
$$

because $\left(S_{t}\right)$ is a martingale $\left(T=\int_{0}^{t} Y_{s}^{2} d s<\infty\right.$ a.s. so the Wald identity $\frac{1}{S_{0}} \mathbb{E}\left(S_{t}\right)=\mathbb{E}\left(e^{W_{T}-\frac{1}{2} T}\right)=1$ holds $($ see Problem 5.7 on page 197 in $[\mathrm{KS} 91])$. We can re-write this identity as $\frac{1}{S_{0}} \mathbb{E}\left(S_{t}-K\right)^{+}=1-\mathbb{E}\left(e^{X_{t}-x_{0}} \wedge e^{k}\right)$. We then just use the dominated convergence theorem as before. To obtain the final answer in terms of the $E_{1}($.$) function, we$ change the variable of integration to $z=\frac{1}{2}\left(y-\sqrt{y^{2}+\sigma^{2}}\right)$.

\subsubsection{Implied volatility}

We now let $\hat{\sigma}_{t}(k)$ denote the Black-Scholes implied volatility of a European put or call option, and let $V(t, k)=\hat{\sigma}_{t}(k)^{2} t$ denote the dimensionless implied variance. The following result shows exactly how $V(t, k)$ tends to infinity as $t \rightarrow \infty$.

Proposition 3.4 For the modified SABR model with $\beta=1$ in $(2), \hat{V}(t, k) \rightarrow \infty$ and $\hat{\sigma}_{t}(k) \rightarrow 0$ as $t \rightarrow \infty$. More precisely, we have the following large-time asymptotic behaviour for the implied variance $\hat{V}(t, k)$ of a put/call option with log-moneyness $k$

$$
\hat{V}(t, k)=4 \log t-4 \log \log t+8 \log (2 \alpha)+8\left(\frac{1}{2} k-\log F(k, 1)\right)+o(1) \quad(t \rightarrow \infty) .
$$

Remark 3.2 Combining the large-time call price estimate in (21) and Theorem 3.1 in Tehranchi[Tehr09], we have

$$
\begin{aligned}
\hat{V}(t, k) & =-8 \log \mathbb{E}\left(S_{t} \wedge K\right)-4 \log \left[-\log \mathbb{E}\left(S_{t} \wedge K\right)\right]+4 k-4 \log \pi+\epsilon(k, t) \\
& =-8 \log \left(\frac{F(k, 1)+o(1)}{\sqrt{2 \pi \alpha^{2} t}}\right)-4 \log \left[-\log \left(\frac{F(k, 1)+o(1)}{\sqrt{2 \pi \alpha^{2} t}}\right)\right]+4 k-4 \log \pi+\epsilon(k, t) \\
& =4 \log t-8 \log F(k, 1)+4 \log \left(2 \pi \alpha^{2}\right)-4 \log \left[-\log (F(k, 1)+o(1))+\frac{1}{2} \log t+\frac{1}{2} \log \left(2 \pi \alpha^{2}\right)\right]+4 k-4 \log \pi+\epsilon(k, t) \\
& =4 \log t-8 \log F(k, 1)+4 \log \left(2 \pi \alpha^{2}\right)-4 \log \left[\frac{1}{2} \log t\left(1-\frac{\log \left(F(k, 1)+o(1)-\log \left(2 \pi \alpha^{2}\right)\right)}{\log (t)}\right)+4 k-4 \log \pi+\epsilon(k, t)\right. \\
& =4 \log t-4 \log \log t+8\left(\frac{1}{2} k-\log F(k, 1)\right)+4 \log \left(4 \alpha^{2}\right)+o(1) \quad(t \rightarrow \infty),
\end{aligned}
$$

where $\epsilon(k, t) \rightarrow 0$ as $t \rightarrow \infty$, which is consistent with (24). 
Remark 3.3 $\hat{V}(t, k)=O(\log t)$ does tend to infinity, but much slower than for the Heston model; under the Heston model, $\hat{\sigma}_{t}^{2}(k)=\hat{V}(t, k) / t$ tends to a positive constant (and also under the Black-Scholes model, trivially). For the modified SABR model here, we need $t=O\left(e^{1 / \epsilon}\right)$ for $\epsilon \ll 1$ for the leading order $4 \log t$ term to be $O(1 / \epsilon)$, and for relative error (which is $O\left(\frac{1}{\log t}\right)$ ) to be $O(\epsilon)$. For this reason, the formula $(24)$ is of more theoretical than practical interest; in practice it is more natural to work directly with the call option asymptotics in Proposition 3.4 .

Remark 3.4 $\frac{1}{2} k-\log F(k, 1)$ is an even function of $k$, so the asymptotic smile $\hat{V}(t, k)$ is symmetric in the log-moneyness $k$ as it should be. $\frac{1}{2} k-\log F(k, 1)$ is the "smile component" of the implied variance.

Proof. We have already shown that $\mathbb{E}\left(S_{t}-K\right)^{+} \nearrow S_{0}$ as $t \rightarrow \infty$. Using (21) and Proposition 2.3 in [FJL10], we know that for all $\delta>0$, there exists a $t^{*}=t^{*}(\delta)$ such that for all $t>t^{*}$

$$
\frac{F(k, 1)}{\sqrt{2 \pi \alpha^{2} t}}(1-\delta) \leq 1-\frac{1}{S_{0}} \mathbb{E}\left(S_{t}-K\right)^{+} \leq e^{-\frac{1}{8} \hat{V}(t, k)(1-\delta)} .
$$

Taking logs and multiplying by -1 we have

$$
\log \left(\sqrt{2 \pi \alpha^{2} t}\right)-\log F(k, 1)-\log (1-\delta) \geq-\log \left[1-\frac{1}{S_{0}} \mathbb{E}\left(S_{t}-K\right)^{+}\right] \geq \frac{1}{8} \hat{V}(t, k)(1-\delta)
$$

and from this we obtain

$$
\frac{1}{2}(1+\delta) \log t \geq-\log \left[1-\frac{1}{S_{0}} \mathbb{E}\left(S_{t}-K\right)^{+}\right] \geq \frac{1}{8} \hat{V}(t, k)(1-\delta)
$$

for $t$ sufficiently large, which yields a lower bound for $\hat{V}(t, k)$. Proceeding similarly for the upper bound, we establish the leading order asymptotic behaviour for the implied variance as

$$
\hat{V}(t, k) \sim 4 \log t \quad(t \rightarrow \infty)
$$

(note that the right-hand side is independent of $k$ and all the parameters of the model). Set $V_{0}(k, t)=4 \log t$ and let $\hat{V}(t, k)=\hat{V}_{0}(t)\left[1+\tilde{V}_{1}(t, k)\right]$, where $\tilde{V}_{1}(t, k) \rightarrow 0$ as $t \rightarrow \infty$. Then for any $\delta>0$, there exists a $t^{* *}=t^{* *}(\delta)$ such that for $t>t^{* *}$ we have

$$
\begin{aligned}
& \frac{F(k, 1)}{\sqrt{2 \pi \alpha^{2} t}}(1-\delta) \leq 1-\frac{1}{S_{0}} \mathbb{E}\left(S_{t}-K\right)^{+} \leq \frac{\sqrt{8}}{\sqrt{\pi \hat{V}(t, k)}} e^{-\frac{1}{8} \hat{V}(t, k)+\frac{1}{2} k}(1+\delta) \\
& =\frac{\sqrt{8}}{\sqrt{\pi \hat{V}_{0}(t)\left(1+\tilde{V}_{1}(t, k)\right)}} e^{-\frac{1}{8} \hat{V}_{0}(t)\left(1+\tilde{V}_{1}(t, k)\right)+\frac{1}{2} k}(1+\delta) .
\end{aligned}
$$

Multiplying by $\sqrt{\pi} e^{\frac{1}{8} \hat{V}_{0}(t)}=\sqrt{\pi} \sqrt{t}$, we obtain

$$
\frac{F(k, 1)}{\sqrt{2 \alpha^{2}}}(1-\delta) \leq \frac{\sqrt{8}}{\sqrt{\hat{V}_{0}(t)\left(1+\tilde{V}_{1}(t, k)\right)}} e^{-\frac{1}{8} \hat{V}_{0}(t) \tilde{V}_{1}(t, k)+\frac{1}{2} k}(1+\delta) \leq \frac{\sqrt{8}}{\sqrt{\hat{V}_{0}(t)}} e^{-\frac{1}{8} \hat{V}_{0}(t) \tilde{V}_{1}(t, k)+\frac{1}{2} k}(1+2 \delta),
$$

for $t$ sufficiently large, and re-arranging we obtain

$$
-\frac{8}{\hat{V}_{0}(t)}\left[\log \left[\frac{\sqrt{\hat{V}_{0}(t)}}{\sqrt{8}} \frac{F(k, 1)}{\sqrt{2 \alpha^{2}}} \frac{1-\delta}{1+2 \delta}\right]-\frac{1}{2} k\right]=\frac{8}{\hat{V}_{0}(t)}\left[-\log \left[\frac{\sqrt{\hat{V}_{0}(t)}}{\sqrt{8}}\right]+\frac{1}{2} k-\log \frac{F(k, 1)}{\sqrt{2 \alpha^{2}}}+\log \left[\frac{1+2 \delta}{1-\delta}\right]\right] \geq \tilde{V}_{1}(t, k) .
$$

This yields a lower bound for $\tilde{V}_{1}(t, k)$, and we proceed similarly for the upper bound. Putting everything together we obtain

$$
\begin{aligned}
\hat{V}(t, k) & =\hat{V}_{0}(t)\left[1+\frac{8}{\hat{V}_{0}(t)}\left[-\frac{1}{2} \log \left(\frac{1}{8} \hat{V}_{0}(t)\right)+\frac{1}{2} k-\log \frac{F(k, 1)}{\sqrt{2 \alpha^{2}}}+o(1)\right]\right] \\
& =4 \log t-4 \log \left(\frac{1}{2} \log t\right)+8\left(\frac{1}{2} k-\log \frac{F(k, 1)}{\sqrt{2 \alpha^{2}}}\right)+o(1) \\
& =4 \log t-4 \log \log t+4 \log \left(2 \alpha^{2}\right)-4 \log \left(\frac{1}{2}\right)+8\left(\frac{1}{2} k-\log F(k, 1)\right)+o(1) \quad(t \rightarrow \infty) .
\end{aligned}
$$




\subsection{The modified SABR model: $\beta<1$}

\subsubsection{The stock price density}

Proposition 3.5 For the modified SABR model with $\beta<1$ in (6), we have the following large-time asymptotic behaviour for the stock price density $p_{t}(S)$

$$
\begin{aligned}
V(S, \beta) & =\lim _{t \rightarrow \infty} \sqrt{2 \pi \alpha^{2} t} p_{t}(S) \\
& =\int_{0}^{\infty} p\left(u, S_{0}, S\right) \frac{1}{u} e^{-1 / 2 u} d u \\
& =\frac{2 \sqrt{S_{0}}}{\left(z^{2}+z_{0}^{2}\right) S^{\frac{3}{2}}}|\bar{\beta}| z_{0}^{2}\left(\frac{G(z)}{z^{2}+z_{0}^{2}}\right)^{-\frac{2 \bar{\beta}-1}{2 \beta}}\left(\frac{z^{2}+z_{0}^{2}}{z_{0}^{2} z^{2} \delta^{2}}\right)^{\frac{1}{2 \beta}}\left(\frac{i}{z z_{0} \delta^{2}}\right)^{-\frac{1}{2 \beta}} F\left(\left[\frac{-1+4 \bar{\beta}}{4 \bar{\beta}}, \frac{2 \bar{\beta}-1}{4 \bar{\beta}}\right],\left[\frac{1}{2} \frac{2 \bar{\beta}-1}{\bar{\beta}}\right], \frac{4 z_{0}^{2} z^{2}}{G(z)^{2}}\right) \mid,
\end{aligned}
$$

where $G(z)=z^{2}+z_{0}^{2}+\left(z z_{0}\right)^{2} \bar{\beta}^{2} \delta^{2}, \delta=y_{0} / \alpha, F\left(\left[n_{1}, n_{2}\right],\left[d_{1}\right], z\right)$ is the generalized hypergeometric function and $z, z_{0}$ are defined in Proposition (2.2).

Proof. From (8) we have

$$
\sqrt{2 \pi \alpha^{2} t} p_{t}(S)=\int_{0}^{\infty} p\left(u, S_{0}, S\right) \frac{1}{\sqrt{2 \pi u^{3}}} \int_{-\infty}^{\infty} \cosh (\xi) e^{-(\cosh \xi)^{2} / 2 u-(\xi+i \pi / 2)^{2} / 2 \alpha^{2} t} d \xi d u
$$

Applying the dominated convergence theorem and using Appendix B we obtain

$$
\begin{aligned}
V(S, \beta) & =\lim _{t \rightarrow \infty} \sqrt{2 \pi \alpha^{2} t} p_{t}(S) \\
& =\int_{0}^{\infty} p\left(u, S_{0}, S\right) \frac{1}{\sqrt{2 \pi u^{3}}} \int_{-\infty}^{\infty} \cosh (\xi) e^{-(\cosh \xi)^{2} / 2 u} d \xi d u \\
& =\int_{0}^{\infty} p\left(u, S_{0}, S\right) \frac{1}{u} e^{-1 / 2 u} d u
\end{aligned}
$$

where we have again used the identity in (12). Evaluating the last integral, we arrive at (33).

Proposition 3.6 For the modified SABR model with $\beta<1$ in (6), we have the following large-time asymptotic behaviour for the stock price distribution $p_{t}(S)$

$$
\begin{aligned}
\lim _{t \rightarrow \infty} \sqrt{2 \pi \alpha^{2} t} \mathbb{P}\left(S_{t}>K\right) & =\int_{K}^{\infty} V(S, \beta) d S<\infty, \\
F(K, \beta)=\lim _{t \rightarrow \infty} \sqrt{2 \pi \alpha^{2} t}\left[S_{0}-\mathbb{E}\left(S_{t}-K\right)^{+}\right] & =\int_{-\infty}^{\infty}(S \wedge K) V(S, \beta) d S<\infty
\end{aligned}
$$

Proof. $S_{t}$ has the same law as a CEV process evaluated at an independent time with the same law as $A_{t}$. The CEV process $d S_{t}=\delta S_{t}^{\beta} d W_{t}$ is a martingale, thus $\mathbb{E}\left(S_{t}\right)=S_{0}$ for the modified uncorrelated SABR model as well. The result then follows by the dominated convergence theorem, similar to the proof of Proposition 3.4.

\subsubsection{Implied volatility}

We now let $\hat{\sigma}_{t}(K)$ denote the Black-Scholes implied volatility of a European put or call option with strike $K$, and let $\hat{V}(t, K)=\hat{\sigma}_{t}(K)^{2} t$ denote the dimensionless implied variance. The following result shows exactly how $\hat{V}(t, K)$ tends to infinity as $t \rightarrow \infty$.

Proposition 3.7 For the modified $S A B R$ model with $\beta<1$ in $(6), \hat{V}(t, K) \rightarrow \infty$ and $\hat{\sigma}_{t}(K) \rightarrow 0$ as $t \rightarrow \infty$. More precisely, we have the following large-time asymptotic behaviour for the implied variance $\hat{V}(t, K)$ of a put/call option with strike $K>0$

$$
\hat{V}(t, K)=4 \log t-4 \log \log t+8 \log (2 \alpha)+8\left(\frac{1}{2} \log \frac{K}{S_{0}}-\log \frac{F(K, \beta)}{S_{0}}\right)+o(1) \quad(t \rightarrow \infty)
$$

Remark 3.5 Remark 3.3 also applies here.

Remark 3.6 $8\left(\frac{1}{2} \log \frac{K}{S_{0}}-\log F(S, \beta)\right)$ is the "smile component" of the implied variance.

Proof. The proof is almost identical to the proof of Proposition 3.4, or we can just use Theorem 3.1 in [Tehr09]. 


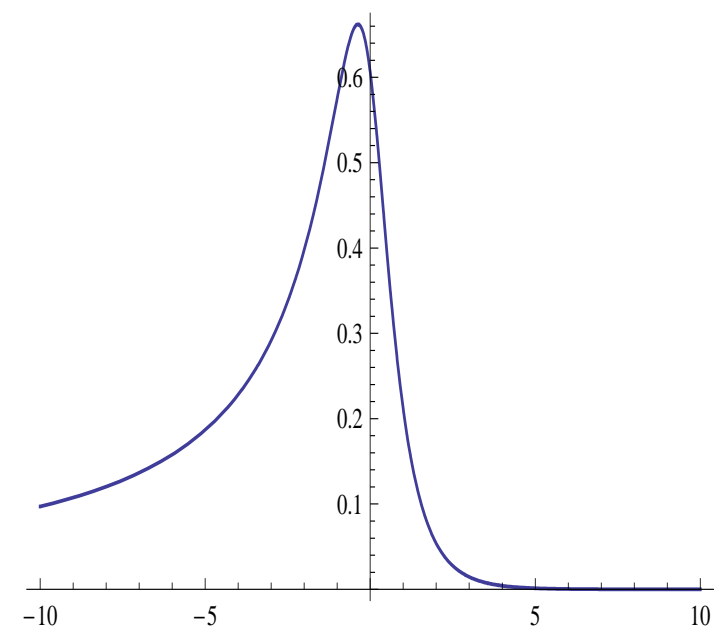

Figure 4: Here we have plotted $V(x, 1)=\lim _{t \rightarrow \infty} \sqrt{2 \pi \alpha^{2} t} p_{t}(x)$ for the modified SABR model with $S_{0}=1, y_{0}=1, \alpha=$ $1, \beta=1$.

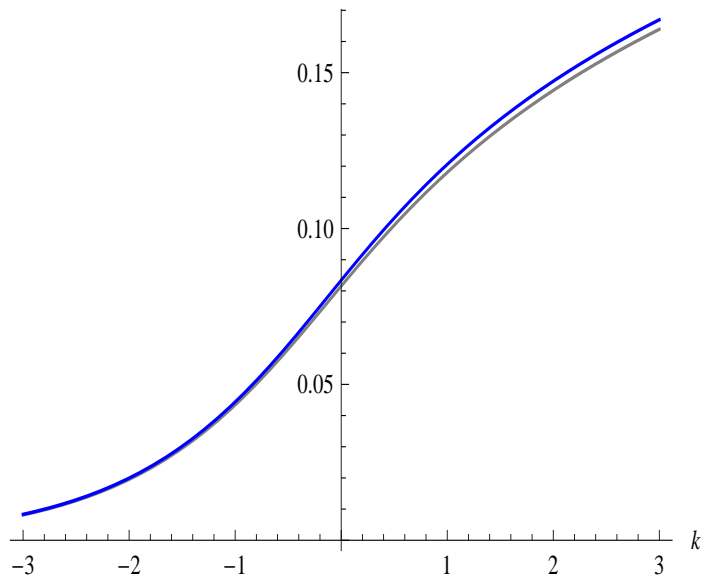

Figure 5: Here we have plotted the true value of $S_{0}-\mathbb{E}\left(S_{t}-K\right)^{+}$(blue) verses the large-time approximation $F(k, 1) / \sqrt{2 \pi \alpha^{2} t}$ (grey) in (21) for $t=30$, as a function of the log-moneyness $k$, for the same model and parameters as the plot above.

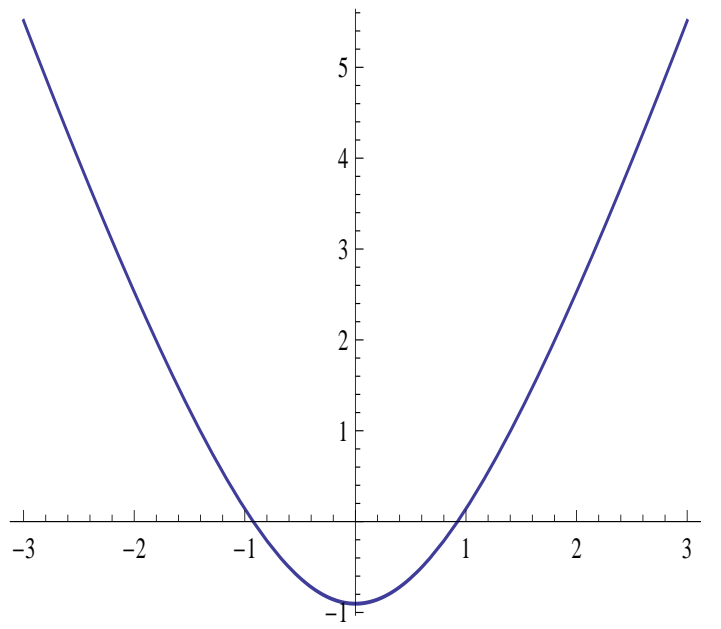

Figure 6: Here we have plotted the smile component $\frac{1}{2} k-\log F(k, 1)$ of the implied variance in $(24)$, which is symmetric in the log-moneyness $k$. 


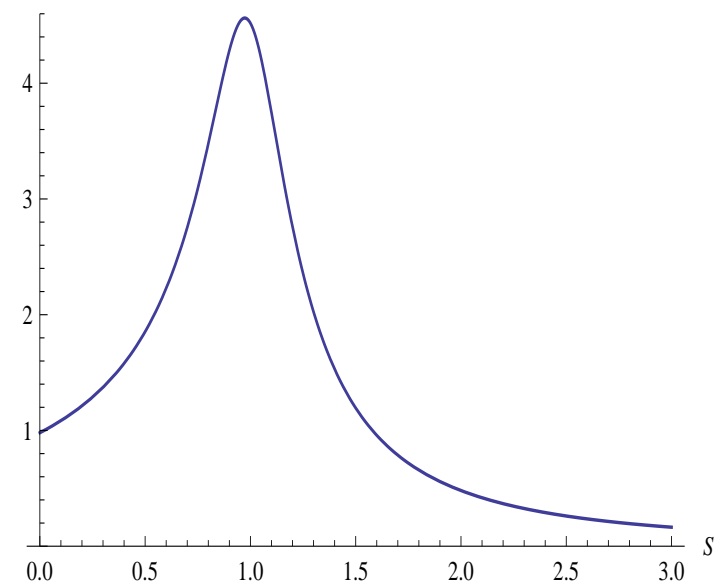

Figure 7: Here we have plotted $V(S, \beta)=\lim _{t \rightarrow \infty} \sqrt{2 \pi \alpha^{2} t} p_{t}(S)$ for the modified SABR model with $S_{0}=1, y_{0}=$ $1, \alpha=1, \delta=.2, \beta=.5$.

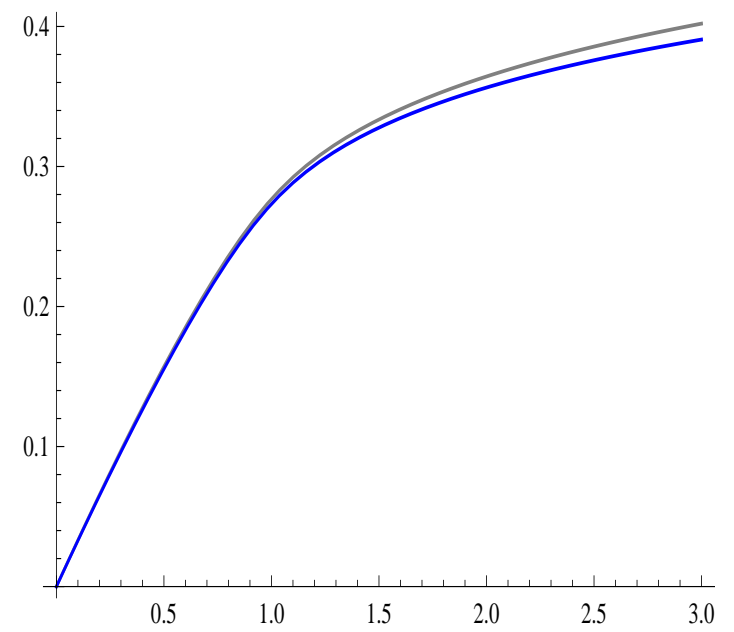

Figure 8: Here we have plotted the true value of $S_{0}-\mathbb{E}\left(S_{t}-K\right)^{+}$(blue) verses the large-time approximation $F(S, \beta) / \sqrt{2 \pi \alpha^{2} t}$ (grey) in (21) for $t=30$, as a function of the strike $K$, for the same model and parameters as the plot above.

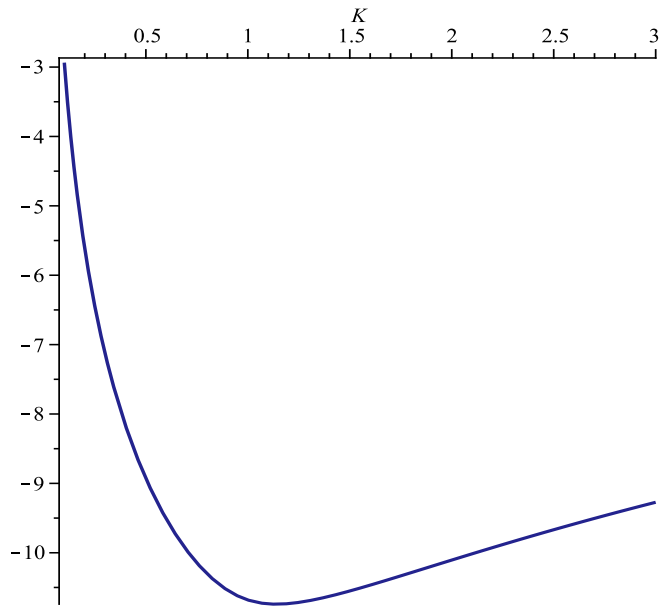

Figure 9: Here we have plotted the smile component $8\left(\frac{1}{2} \log K-\log F(S, \beta)\right)$ of the implied variance in (35), which is negatively skewed as we would expect, because $\beta<1$. 


\section{References}

[AS72] Abramowitz, M. and I.Stegun, Handbook of Mathematical Functions with Formulas, Graphs, and Mathematical Tables, New York: Dover Publications, 1972.

[AG97] Alili, L. and Gruet, J.-C., "An explanation of a generalized Bougerols identity in terms of hyperbolic Brownian motion", in , 1997.

[CMY98] Comtet, A., Monthus, C. and Yor, M., "Exponential functionals of Brownian motion and disordered systems", J. Appl. Prob., 35, 255-271. MR1641852, 1998.

[Cox75] Cox, J. (1975), "Notes on option pricing I: Constant elasticity of variance diffusions" working paper, Stanford university, reprinted in Journal of Portfolio Management, 22, 15-17, 1996.

[DavLin01] Davydov, D. and V. Linetsky, "The Valuation and Hedging of Barrier and Lookback Options under the CEV Process," Management Science, 47, pgs 949-965, 2001.

[Forde09] Forde, M., "Small-time asymptotics for a general local-stochastic volatility model, using the heat kernel expansion", 2009 (submitted).

[FJM09] Forde, M., A.Jacquier and A.Mijatovic, "Asymptotic formulae for implied volatility in the Heston model", forthcoming in the Proceedings of the Royal Society A.

[FJ09] Forde, M. and A.Jacquier, "The Large-maturity smile for the Heston model", 2009, forthcoming in Finance and Stochastics.

[FJL10] Forde, M., A.Jacquier and R.Lee, "Small-time asymptotics for implied volatility under the Heston model: Part 2", submitted 2010.

[GS06] Gulisashvili, A. and E.M.Stein, "Asymptotic Behavior of the Distribution of the Stock Price in Models with Stochastic Volatility: The Hull-White Model", C. R. Acad Sci. Paris, Ser. I 343, 519-523, 2006

[GS07] Gulisashvili, A. and E.M.Stein, "Asymptotic behavior of distribution densities in models with stochastic volatility, I ", forthcoming in Mathematical Finance.

[HL09] Henry-Labordère, P., "Analysis, Geometry, and Modeling in Finance: Advanced Methods in Option Pricing", Chapman \& Hall (2008).

[Isl09] Islah, O., "Solving SABR in exact form and unifying it with LIBOR market model", working paper, 2009.

[KS91] Karatzas, I. and S.Shreve, "Brownian motion and Stochastic Calculus", Springer-Verlag, 1991.

[Laur08] Laurence, P., "Implied Volatility, Fundamental solutions, asymptotic analysis and symmetry methods", Cal Tech, April 2008.

[Lew00] Lewis, A., "Option Valuation Under Stochastic Volatility: With Mathematica Code", Finance Press, 2000.

[MY05] Matsumoto, H. and Yor, M., "Exponential Functionals of Brownian motion I: Probability laws at fixed time, Probab. Surveys, 2, pgs 312-347, 2005.

[1] Paulot, L., "Asymptotic Implied Volatility at the Second Order With Application to the SABR Model", working paper (2009).

[Tehr09] Tehranchi, M., "Asymptotics of implied volatility far from maturity", Journal of Applied Probability, 46 (3), 629-650 (2009). 


\section{A Use of Fubini's theorem for $\beta=1$}

Here we justify the use of Fubini's theorem in the proof of Proposition 2.1. For $t>1$ we have

$$
\begin{aligned}
I & =\int_{0}^{\infty} \frac{1}{y_{0} \sqrt{2 \pi u}} e^{-\left(x+\frac{1}{2} u y_{0}\right)^{2} / 2 u y_{0}} \frac{1}{\sqrt{2 \pi u^{3}}} \int_{-\infty}^{\infty} \cosh (\xi) e^{-(\cosh \xi)^{2} / 2 u-\left(\xi^{2}-\pi^{2} / 4\right) / 2 t} d \xi d u \\
& \leq \int_{0}^{\infty} \frac{1}{y_{0} \sqrt{2 \pi u}} e^{-\left(x+\frac{1}{2} u y_{0}\right)^{2} / 2 u y_{0}} \frac{1}{\sqrt{2 \pi u^{3}}} e^{\frac{\pi^{2}}{8 t}} \int_{-\infty}^{\infty} \cosh (\xi) e^{-(\cosh \xi)^{2} / 2 u} d \xi d u \\
& =e^{\frac{\pi^{2}}{8 t}} \int_{0}^{\infty} \frac{1}{y_{0} \sqrt{2 \pi u}} e^{-\left(x+\frac{1}{2} u y_{0}\right)^{2} / 2 u y_{0}} \frac{1}{u} e^{-1 / 2 u} d u \\
& =e^{\frac{\pi^{2}}{8 t}} \frac{1}{\sqrt{x^{2}+y_{0}^{2}}} e^{-\frac{1}{2}\left(x+\sqrt{x^{2}+y_{0}^{2}}\right)}<\infty
\end{aligned}
$$

\section{B Use of Fubini's theorem for $\beta<1$}

- Here we justify the use of Fubini's theorem in the proof of Proposition 2.2. For $S<1, t>1$ we have the following upper bound for the CEV density

$$
p\left(t, S_{0}, S\right) \leq \frac{S^{-2 \bar{\beta}-\frac{3}{2}} S_{0}^{\frac{1}{2}}}{\delta^{2}|\bar{\beta}| t} \exp \left(-\frac{S^{2}}{2 \delta^{2} \bar{\beta}^{2} t}\right) I_{\nu}\left(\frac{S_{0}^{-\bar{\beta}} S^{-\bar{\beta}}}{\delta^{2} \bar{\beta}^{2}}\right) .
$$

Then

$$
\begin{aligned}
I & =\int_{0}^{\infty} p\left(u, S_{0}, S\right) f\left(u, \alpha^{2} t\right) d u \\
& \leq e^{\frac{\pi^{2}}{8 t}} \frac{S^{-2 \bar{\beta}-\frac{3}{2}} S_{0}^{\frac{1}{2}}}{\delta^{2}|\bar{\beta}|} I_{\nu}\left(\frac{S_{0}^{-\bar{\beta}} S^{-\bar{\beta}}}{\delta^{2} \bar{\beta}^{2}}\right) \int_{0}^{\infty} \frac{1}{u} \exp \left(-\frac{S^{2}}{2 \delta^{2} \bar{\beta}^{2} u}\right) \frac{1}{\sqrt{2 \pi u^{3}}} \int_{-\infty}^{\infty} \cosh (\xi) e^{-(\cosh \xi)^{2} / 2 u} d \xi d u \\
& \leq e^{\frac{\pi^{2}}{8 t}} \frac{S^{-2 \bar{\beta}-\frac{3}{2}} S_{0}^{\frac{1}{2}}}{\delta^{2}|\bar{\beta}|} I_{\nu}\left(\frac{S_{0}^{-\bar{\beta}} S^{-\bar{\beta}}}{\delta^{2} \bar{\beta}^{2}}\right) \int_{0}^{\infty} \frac{1}{u} \exp \left(-\frac{S^{2}}{2 \delta^{2} \bar{\beta}^{2} u}\right) \frac{1}{u} e^{-1 / 2 u} d u \\
& <\infty .
\end{aligned}
$$

- For $S>1, t>1$ we have the following upper bound for the CEV density

$$
p\left(t, S_{0}, S\right) \leq \frac{S^{-2 \bar{\beta}-\frac{3}{2}} S_{0}^{\frac{1}{2}}}{\delta^{2}|\bar{\beta}| t} \exp \left(-\frac{(\log S)^{2}}{2 \delta^{2} \bar{\beta}^{2} t}\right) I_{\nu}\left(\frac{S_{0}^{-\bar{\beta}} S^{-\bar{\beta}}}{\delta^{2} \bar{\beta}^{2}}\right) .
$$

Using this inequality as in previous case, we find that

$$
I=\int_{0}^{\infty} p\left(u, S_{0}, S\right) f\left(u, \alpha^{2} t\right) d u<\infty .
$$

